\title{
Modeling the Monthly Variations of Urban Heat Island in Beijing City with MODIS Surface Products
}

\author{
Ji Zhou ${ }^{12}$ \\ 1. Jiangsu Key Laboratory of Resources and Environmental \\ Information Engineering, China University of Mining and \\ Technology \\ Xuzhou, China \\ jzhou233@uestc.edu.cn
}

\author{
Xu Zhang ${ }^{2}$ \\ 2. School of Resources and Environment, University of \\ Electronic Science and Technology of China \\ Chengdu, China \\ xuzhang@uestc.edu.cn
}

\begin{abstract}
As a climatic phenomenon, the urban heat island (UHI) obtains intrinsic variation characteristics at different temporal scales. However, investigations on the intra-annual variations of UHI in mega cities are still rare. In this study, highquality LST time-series datasets of the Beijing municipality are generated from MODIS (Moderate-resolution Imaging Spectroradiometer) surface products, for investigating the monthly variations of UHI in Beijing city. These time-series datasets cover the periods of 2001-2008 (for 10:30 and 22:30 local solar time) and 2003-2008 (for 13:30 and 01:30 local solar time). Results demonstrate that the daytime UHI effect of Beijing city was most significant in the summer (August), while it was weakest in the winter (January and February). Generally speaking, there were urban heat sink phenomena in the city of Beijing in winter or March, but the city appeared as heat islands in conditions with snowfalls. The UHI intensity in the afternoon (13:30 local time) was commonly higher than that in the morning (10:30 local time). For the nighttime UHI of Beijing, it was most significant in the winter (January) and weakest in the summer (July). The UHI intensity in the middle-night was commonly lower than that in the evening. The monthly variations of UHI of Beijing city are related with the climatic background, meteorological conditions, and surface characteristics.
\end{abstract}

Keywords-urban heat island; land surface temperature; time series; monthly variations; MODIS; Beijing

\section{INTRODUCTION}

Rapid urbanization of many world cities has caused land use and land cover changes and induced significant anthropogenic influences on climate ${ }^{[1]}$. One of the most wellknown climatic effects induced by urbanization is the urban heat island (UHI) effect. The heat-waves caused by the UHI effect influence the human heaths, quality of life, and energy consumption. Satellite remote sensing obtains many advantages for monitoring the UHI effects. In the past four decades, remote sensing of UHI has been well documented through topics such as the spatial structures and patterns of surface UHI ${ }^{[2]}$. However, advances about temporal variations of UHI have been slowed down due to the low temporal resolutions of thermal remote sensing images or the estimated land surface temperatures (LSTs). Findings about the intraannual variations of UHI are still extremely rare. This has become an important limitation for quantitative analysis of UHI. The objectives of this study are to model the monthly variations of UHI of Beijing city and quantify the differences between UHIs in different seasons by using the MODIS surface products.

\section{STUDY AREA AND DATASETS}

\section{A. Study Area}

The city of Beijing, China, has been chosen as the study area. There are mountains to the west, to the north, and to the northeast of the city. The altitudes of the plain regions in the study area are 20 60 $\mathrm{m}$ above sea level. Beijing has distinct seasons, with a hot and humid summer and a cold and dry winter. There are currently six ring roads in Beijing, and the main city is surrounded by the Sixth Ring Road. The dominating land cover in the rural areas at a similar altitude as the city is cropland. These rural areas are covered by abundant crops in the summer, and they turn to bare soil or are sparsely vegetated in the winter ${ }^{[3]}$.

\section{B. Datasets}

MODIS provides LSTs of the globe, including the monthly composited MOD11C3/MYD11C3 product with a $0.05^{\circ}$ spatial resolution and the daily MOD11A1/MYD11A1 product with a $1 \mathrm{~km}$ spatial resolution. However, the MOD11A1/MYD11A1 product appears to suffer the errors of the MODIS cloud-mask algorithm: there are less valid LSTs in winter in all regions, especially in the city of Beijing, due to clouds and heavy air pollution (Wan Zhengming, personal communication, 28 July 2009). Therefore, these two categories of products are combined to generate four high-quality monthly LST timeseries datasets. The datasets used in this study is shown in Table I.

TABLE I. REMOTELY SENSED DATA USED FOR MODELING THE MONTHLY VARIATIONS OF THE UHIS

\begin{tabular}{|c|c|c|c|}
\hline Product & Parameter & Duration & Local time \\
\hline MOD11A1 & Daily LST & $2001.01-2008.12$ & $10: 30,22: 30$ \\
\hline MYD11A1 & Daily LST & $2003.01-2008.12$ & $13: 30,01: 30$ \\
\hline MOD11C3 & Monthly LST & $2001.01-2008.12$ & $10: 30,22: 30$ \\
\hline MYD11C3 & Monthly LST & $2003.01-2008.12$ & $13: 30,01: 30$ \\
\hline MOD13A2 & 16-day vegetation indices & $2001.01-2008.12$ & $10: 30$ \\
\hline MOD09A2 & 8-day surface reflectance & 2001.01-2008.12 & $10: 30$ \\
\hline
\end{tabular}

\section{METHODOLOGY}

\section{A. Generating Monthly LST Time Series}

The approach for generating the monthly LST time series can be divided into two stages. In the first stage, the daily 
MOD11A1/MYD11A1 products in each month are grouped, and the number of good observations of each pixel in each month is calculated. If the number of good observations of a pixel during a month is not less than three, then the mean LST of these good observations is recorded as the mean LST of the corresponding pixel in this month.

Examinations on the monthly composited LST of the Beijing municipality based on the MODIS daily LST products (i.e. MOD11A1 and MYD11A1 products) reveal that these are too many pixels located in the urban areas cannot obtain even one clear observations during the daytime in the winter and early spring. The period spans from December to March in the next year. These LST data are treated in the second stage. The monthly LST images, in which the number of pixels those obtained less than three good observations during a month, are flagged. Then the entire flagged LST images are sharpened from the corresponding MOD11C1 and MYD11C1 LST products with a disaggregating procedure developed by [4]. In this procedure, the LST and NDVI (Normalized Difference Vegetation Index) with coarser spatial resolution (0.05 degree) and NDVI with higher spatial resolution $(1 \mathrm{~km})$ are combined to estimate the LST with a $1-\mathrm{km}$ spatial resolution. An assumption of this procedure is that the relationship of LSTNDVI at coarser resolution (e.g. 0.05 degree) can be extended to finer resolution (e.g. $1 \mathrm{~km})$.

\section{B. Identification of the UHIs}

A straightforward spatial analysis approach is used here to identify the surface UHIs of Beijing. Firstly, the urban surfaces are extracted with a simple threshold method based on NDVI, which is calculated from the surface reflectance derived from the MOD09A1 products in the summer. Secondly, the rural pixels are extracted. All the rural pixels located outside the Sixth Ring Road and with altitude lower than $100 \mathrm{~m}$ are selected as the rural pixels. The mean land surface temperature of the rural pixels is used as the base temperature. Thirdly, the UHI signatures are calculated by subtracting the based temperature from the land surface temperatures of all pixels with altitude lower than $100 \mathrm{~m}$. The mean value of the UHI signatures of all the urban pixels located within the Sixth Ring Road is calculated and used as the UHI intensity of Beijing. As a matter of fact, a negative UHI intensity reveals that the urban heat sink (UHS) phenomenon appears in the city.

\section{RESULT AND DISCUSSION}

\section{A. Evaluating the Monthly LST Time Series}

The extracted monthly mean rural surface temperatures (RST) are compared with the air temperature of $1.5 \mathrm{~m}$ above the ground measured at the Beijing Meteorological Observatory. Although the monthly rural surface temperature (RST) and the monthly air temperature are different, they should have similar intra-annual variations. Generally speaking, the maximum air temperature often appears at around 14:0015:00 local time in the afternoon, and the minimum air temperature often appears before the dawn. Therefore, the RSTs at 13:30 local time are compared with the maximum air temperatures, and the RSTs at 01:30 local time are compared with the minimum air temperatures. Although the overpass times of Terra satellite, i.e. 10:30 and 22:30 local time, are not corresponding to the times with minimum or maximum air temperature, the RSTs at 10:30 are compared with the maximum air temperature and the RSTs at 22:30 are compared with the minimum air temperature, for examining their intraannual trends. Some of the scatter-plots between the monthly RSTs and air temperatures are shown in Fig. 1. Significant linear relationships between the RSTs and air temperatures are found. The daytime LSTs correspond to the maximum air temperature, while the nighttime LSTs correspond to the minimum air temperature. These linear relationships demonstrate that the RST and air temperature have similar intra-annual variations. Thus, it is applicable to use the generated LST time series datasets to examine the monthly UHI variations.
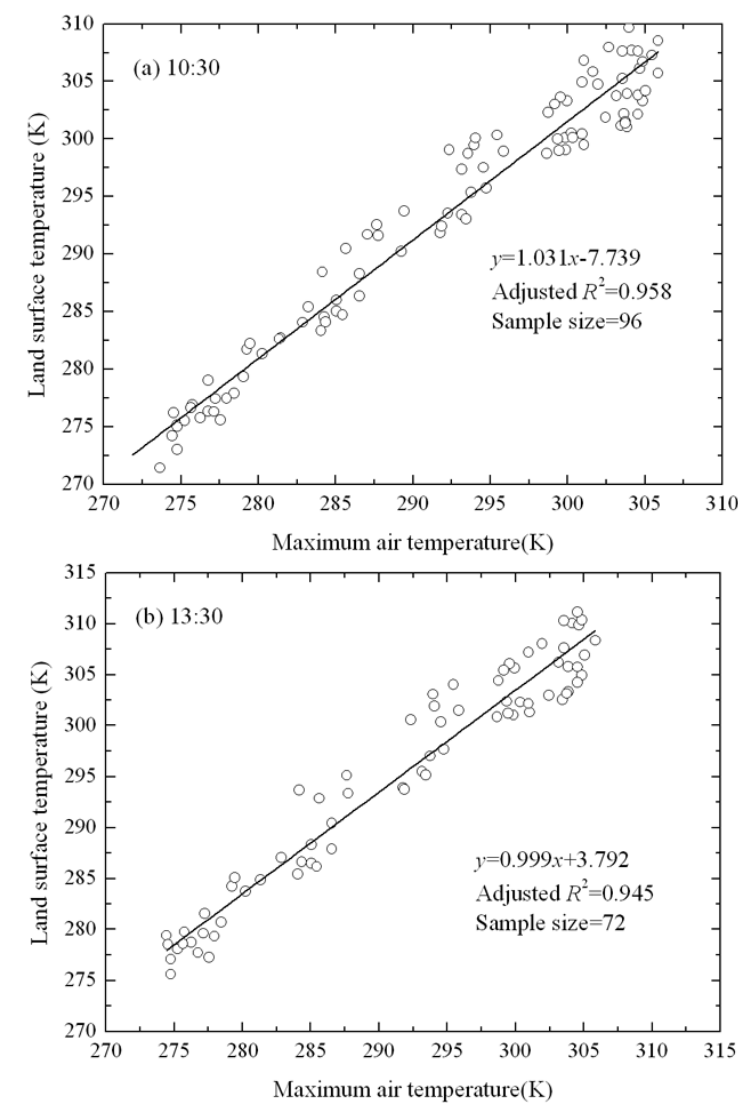

Figure 1. Comparisons between the monthly air temperature measured at $\mathrm{BMO}$ and the rural surface temperature derived from the monthly LST time series datasets.

\section{B. Monthly Variations of Daytime UHI}

The monthly UHI intensities of Beijing city at 10:30 local time from 2001 to 2008 are shown in Fig. 2a, and those at 13:30 local time from 2003 to 2008 are shown in Fig. 2b. The daytime UHI intensity has evident intra-annual periods. It is straightforward to understand that because the LST obtains the intra-annual periods. Generally speaking, there were daytime UHI effects in Beijing city in most months in a year. Taking 
the entire city as a whole, the UHIs in August were the most significant, revealing by the monthly variations of UHIs observed by Terra and Aqua satellites. The UHI intensities in the winter and early spring (i.e. December, January, February, and March,) in different years might possess different signs, though the absolute values of them are small. For example, the daytime UHI intensities of February were positive in 2003, 2005, and 2006, while they were negative in 2004, 2007, and 2008. Literatures suggested that the city of Beijing obtains the urban heat sink effect in the winter ${ }^{[3,5]}$, due to urban and rural differences in vegetation abundance, albedo, and thermal inertia. Comparison between Fig. 2a and Fig. 2b indicates that the UHI intensities in the morning (10:30 local time) were generally lower than those in the afternoon (13:30 local time). The monthly UHI intensities at 10:30 in August were commonly around $5.0 \mathrm{~K}(4.6 \mathrm{~K} \sim 5.6 \mathrm{~K}$, with the mean value and standard deviation as $5.1 \mathrm{~K}$ and $0.3 \mathrm{~K}$, respectively), while those at 13:30 in August were commonly around $6.0 \mathrm{~K}(5.2 \mathrm{~K} \sim 6.6 \mathrm{~K}$, with the mean value and standard deviation as $6.0 \mathrm{~K}$ and $0.5 \mathrm{~K}$, respectively).

The monthly mean UHI intensities at 10:30 from 2001 to 2008 and the monthly mean UHI intensities at 13:30 from 2003 and 2008 are calculated. Statistics confirms the finding that the UHI was the most significant in August; while the UHIs in the winter, i.e. December, January, and February, were the weakest (with the mean intensities around $0 \mathrm{~K}$ ). The daytime UHI intensities in July and September were about $1.0 \mathrm{~K}-1.5 \mathrm{~K}$ lower than those in August. For instance, the mean UHI intensities in July was $3.6 \mathrm{~K} \pm 0.9 \mathrm{~K}$ at $10: 30$ local time and $5.0 \mathrm{~K} \pm 1.0 \mathrm{~K}$ at 13:30 local time, while the mean UHI intensities in September was $3.6 \mathrm{~K} \pm 0.5 \mathrm{~K}$ at $10: 30$ and $4.5 \mathrm{~K} \pm 0.2 \mathrm{~K}$ at $13: 30$.

With the monthly UHI intensities, the seasonal UHI intensities are further calculated. The 12 months are divided into four seasons following: (1) the spring: March, April, and May; (2) the summer: June, July, and August; (3) the autumn: September, October, and November; and (4) the winter: December, January, and February. The calculated mean seasonal daytime UHI intensities at 10:30 and 13:30 are shown in Table II. It is apparent that the city obtained the most significant UHI effect in the summer, while it obtained the weakest UHI effect in the winter. Except in the winter, the UHI effect occurred in the afternoon was more significant than that appeared in the morning in all seasons.

TABLE II. THE SEASONAL DAYTIME UHI INTENSITIES OF BEIJING CITY.

\begin{tabular}{|c|c|c|c|c|}
\hline Local time & Spring & Summer & Autumn & Winter \\
\hline $10: 30$ & $1.3 \mathrm{~K} \pm 1.1 \mathrm{~K}$ & $3.6 \mathrm{~K} \pm 1.3 \mathrm{~K}$ & $1.8 \mathrm{~K} \pm 1.4 \mathrm{~K}$ & $0.1 \mathrm{~K} \pm 0.6 \mathrm{~K}$ \\
\hline $13: 30$ & $1.7 \mathrm{~K} \pm 1.0 \mathrm{~K}$ & $4.5 \mathrm{~K} \pm 1.6 \mathrm{~K}$ & $2.2 \mathrm{~K} \pm 1.7 \mathrm{~K}$ & $0.1 \mathrm{~K} \pm 0.6 \mathrm{~K}$ \\
\hline
\end{tabular}
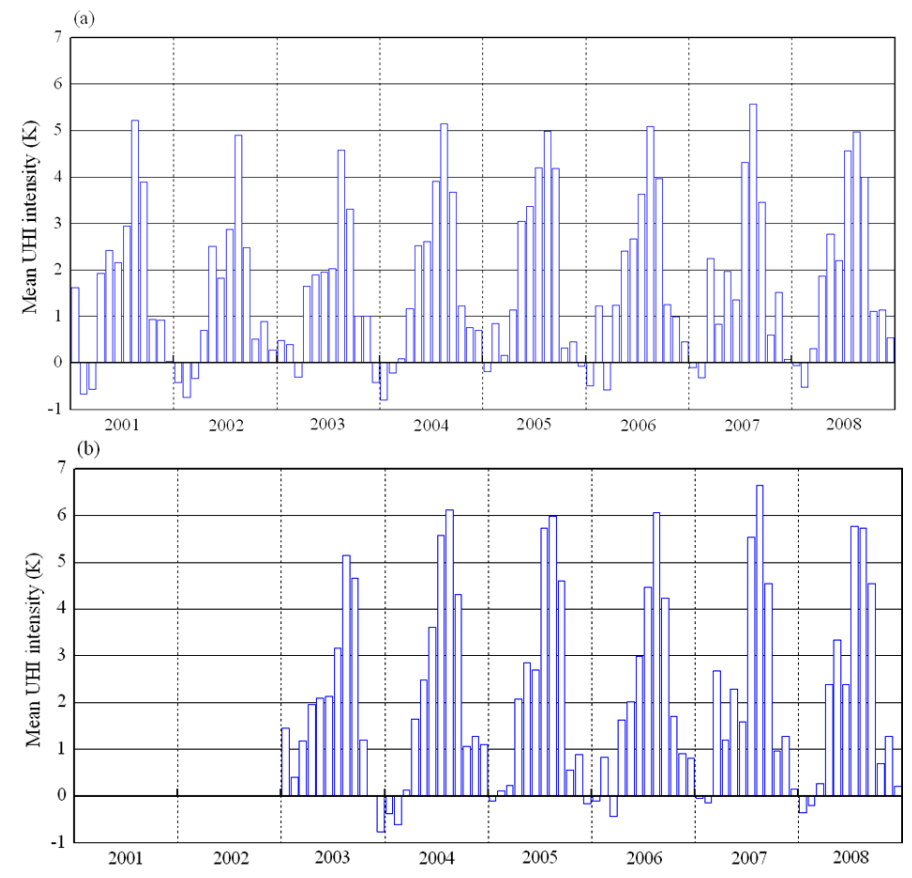

Figure 2. The monthly daytime UHI intensities of Beijing city in each month. (a) 10:30 local time from 2001 to 2008; (b) 13:30 local time from 2003 to 2008 .

\section{Monthly Variations of Nighttime UHI}

The monthly UHI intensities of Beijing at 22:30 local time from 2001 to 2008 are shown in Fig. 3, and those at 01:30 local time from 2003 to 2008 are shown in Fig. 4. Compared to the daytime UHI intensity, the intra-annual periods of the nighttime UHI intensity was not evident. Taking the entire city as a whole, there were nighttime UHI effects in all months, and this finding corresponds to the findings reported by other researches about nighttime UHI in the city of Beijing ${ }^{[5,6]}$. In general, the nighttime UHI intensities in early spring or winter were higher than those in other months. In order to compare the monthly UHI intensities, the mean monthly UHI intensities at 22:30 from 2001 to 2008 and those at 01:30 from 2003 to 2008 are calculated. The result demonstrates that the nighttime UHI intensity of Beijing had inverse intra-annual variation compared with the daytime UHI intensity. The nighttime UHI was the most significant in January; while those in February and December were lower. For instance, the mean UHI intensities in January was $3.6 \mathrm{~K} \pm 0.6 \mathrm{~K}$ at $22: 30$ local time and $3.5 \mathrm{~K} \pm 0.4 \mathrm{~K}$ at 01:30 local time; while the mean UHI intensities in February and December were $3.4 \mathrm{~K} \pm 0.7 \mathrm{~K}$ and $3.2 \mathrm{~K} \pm 0.3 \mathrm{~K}$ at $22: 30$ local time, respectively, and those were $3.0 \mathrm{~K} \pm 0.6 \mathrm{~K}$ and $2.9 \mathrm{~K}$ and $0.5 \mathrm{~K}$ at $01: 30$ local time, respectively. The nighttime UHI was the weakest in August, in which the mean monthly UHI intensity was $2.3 \mathrm{~K} \pm 0.3 \mathrm{~K}$ at $22: 30$ and $2.1 \mathrm{~K} \pm 0.2 \mathrm{~K}$ at $01: 30$.

With the monthly nighttime UHI intensities, the seasonal nighttime UHI intensities are further calculated. The calculated mean seasonal daytime UHI intensities at 10:30 and 13:30 are shown in Table III. It is apparent that the city obtained the most significant nighttime UHI effect in the winter, while it obtained the weakest nighttime UHI effect in the summer. The nighttime 
UHI intensities at 01:30 were about $0.2 \mathrm{~K} \sim 0.4 \mathrm{~K}$ lower than those at 22:30. With the monthly nighttime UHI intensities, the seasonal nighttime UHI intensities are further calculated. The calculation result shows that the city obtained the most significant nighttime UHI effect in the winter, while it obtained the weakest nighttime UHI effect in the summer. The nighttime UHI intensities at 01:30 were about $0.2 \mathrm{~K} \sim 0.4 \mathrm{~K}$ lower than those at 22:30.
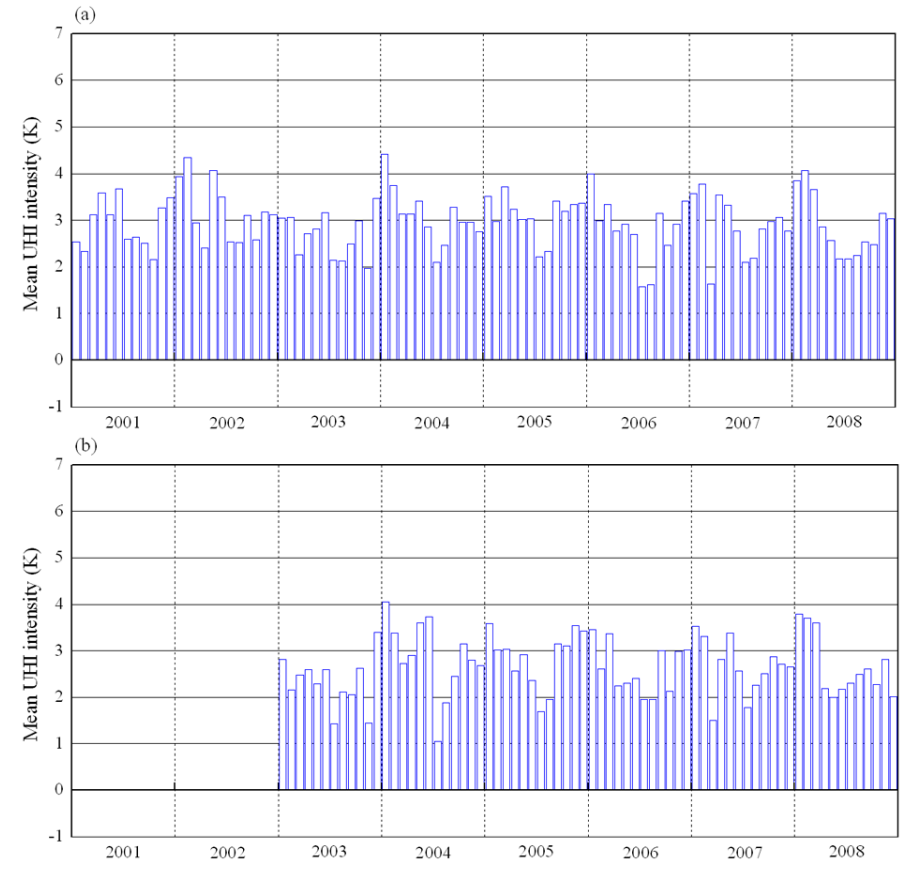

Figure 3. The monthly nighttime UHI intensities of Beijing city in each month. (a) 22:30 local time from 2001 to 2008; (b) 01:30 local time from 2003 to 2008 .

TABLE III. The SEASONAL NIGHTTIME UHI INTENSITIES OF BEIJING.

\begin{tabular}{|c|c|c|c|c|}
\hline Local time & Spring & Summer & Autumn & Winter \\
\hline $22: 30$ & $3.1 \mathrm{~K} \pm 0.5 \mathrm{~K}$ & $2.5 \mathrm{~K} \pm 0.5 \mathrm{~K}$ & $2.9 \mathrm{~K} \pm 0.4 \mathrm{~K}$ & $3.4 \mathrm{~K} \pm 0.6 \mathrm{~K}$ \\
\hline $01: 30$ & $2.7 \mathrm{~K} \pm 0.6 \mathrm{~K}$ & $2.1 \mathrm{~K} \pm 0.6 \mathrm{~K}$ & $2.7 \mathrm{~K} \pm 0.5 \mathrm{~K}$ & $3.1 \mathrm{~K} \pm 0.6 \mathrm{~K}$ \\
\hline
\end{tabular}

\section{CONCLUSIONS}

In this research, four high-quality land surface temperature (LST) time-series datasets at 10:30, 13:30, 22:30, and 01:30 local time, in the Beijing municipality are generated based on the daily MODIS LST products with a 1-km spatial resolution, the monthly .MODIS LST products with a 0.05 -degree spatial resolution, and the NDVI data derived from the 16-day MODIS vegetation index products with a $1-\mathrm{km}$ spatial resolution. With these four LST datasets, the monthly UHI signatures of Beijing are quantified.
Evaluations of the LST time series demonstrate that the generated LST time series possess good accuracies for examining the monthly variations of UHI in the city of Beijing. Statistics reveal that the daytime urban heat island effect was most significant in the summer (August), while it was weakest in the winter (January and February). Generally speaking, there were urban heat sink phenomena in the city of Beijing in winter or March, but the city appeared as heat islands in conditions with snowfalls. The UHI intensity in the afternoon (13:30 local time) was commonly higher than that in the morning (10:30 local time). For the nighttime UHI of Beijing, it was most significant in the winter (January) and weakest in the summer (July). The UHI intensity in the middle-night was commonly lower than that in the evening.

\section{ACKNOWLEDGMENT}

This work was supported in part by the Scientific Research Foundation of Jiangsu Key Laboratory of Resources and Environmental Information Engineering (China University of Mining and Technolgoy) (No. JS201103), by the National Natural Science Foundation of China (grant number: 41101380 and 41071258), and by the Postdoctoral Science Foundation of China (grant number: 2011M500145). The MODIS surface products were provided by the National Aeronautics and Space Administration. The meteorological datasets were provided by China Meteorological Data Sharing Service System.

\section{REFERENCES}

[1] L. Zhou, R. E. Dickinson, Y. H. Tian, et al., "Evidence for a significant urbanization effect on climate in China," Proc. Natl. Acad. Sci. USA, vol. 101, pp. 9540-9544, June, 2004.

[2] J. A. Voogt, and T. R. Oke, "Thermal remote sensing of urban climates," Remote. Sens. Environ, vol. 86, pp. 370-384, August, 2003.

[3] J. Zhou, D. Y. Hu, Q. H. Weng, "Analysis of radiation budget during the summer and winter in the Beijing metropolitan area, China," J. Appl. Remote. Sens, vol. 4, January, 2010, doi: 10.1117/1.3374329.

[4] W. P. Kustas, J. M. Norman, M. C. Anderson, et al., "Estimating subpixel surface temperatures and energy fluxes from the vegetation index-radiometric temperature relationship," Remote. Sens. Environ, vol. 85, pp. 429-440, June, 2003.

[5] K. Wang, J. Wang, P. Wang, et al., "Influences of urbanization on surface characteristics as derived from the Moderate-Resolution Imaging Spectroradiometer: A case study for the Beijing metropolitan area," J. Geophys. Res, vol. 112, August, 2007, doi: 10.1029/2006JD007997.

[6] J. Zhou, Y. Chen, J. Wang, et al., "Maximum nighttime urban heat island (UHI) intensity simulation by integrating remotely sensed data and meteorological observations," IEEE J. Sel. Top. Appl. Earth. Obs, vol. 4, pp. 138-146, March, 2011. 